\title{
Endometriosis-associated dyspareunia: the impact on women's lives
}

\author{
Elaine Denny, Christopher H Mann
}

\begin{abstract}
Background and methodology Endometriosis is a chronic condition in which endometrial glands and stroma are present outside of the uterus. Whereas chronic pelvic pain is the most commonly experienced pain of endometriosis, many women also suffer from deep dyspareunia. In order to determine how much of an impact endometriosis-associated dyspareunia has on the lives and relationships of women a qualitative study using semi-structured interviews, supplemented with quantitative data on the extent of dyspareunia, was conducted in a dedicated endometriosis clinic in the West Midlands, UK with 30 women aged from 19 to 44 years.
\end{abstract}

Results The main outcome measures were the extent of dyspareunia within the sample of women, and the impact of dyspareunia on quality of life. The experience of dyspareunia was found to be higher than in previous research. Three main themes emerged. The experience of

\section{Introduction}

Endometriosis is a chronic condition in which endometrial glands and stroma are present outside of the uterus. This abnormal tissue is most commonly, but not exclusively, found in the abdominal cavity. The cyclical hormonal influence of progesterone and oestrogen on these endometrial deposits causes activation of the tissue, leading to the typical symptom of pain. ${ }^{1}$ The frequency of diagnosis of endometriosis has increased over the last 15-20 years. ${ }^{2}$ However, this may not reflect a rise in the true incidence of the condition, rather in the methodology employed in diagnosing the condition, as this increase in the number of cases diagnosed coincided with the introduction of laparoscopy for the direct confirmation of the presence of endometrial tissue in the abdomen. ${ }^{3}$ It is also thought that later and less frequent childbearing, with a consequent increase in menstrual cycles, theoretically presents more opportunity for endometrial tissue to implant outside the uterus. ${ }^{2}$

The true prevalence of this disease is unknown, but it is usually estimated to be present in between $5 \%$ and $15 \%$ of women of reproductive age. ${ }^{2}$ Whereas chronic pelvic pain is the most commonly experienced type of pain in endometriosis, many women also suffer from deep dyspareunia ${ }^{4}$ (deep pelvic pain associated with sexual intercourse). The symptom of dyspareunia has

\section{Faculty of Health, University of Central England, Birmingham, UK \\ Elaine Denny, PhD, Head of Health Policy and Public Health Division \\ Birmingham Women's Hospital, Birmingham, UK Christopher H Mann, MD, Senior Lecturer in Gynaecological Oncology}

Correspondence to: Professor Elaine Denny, Department of Community Health and Social Work, Health Policy and Public Health Division, University of Central England, Perry Barr, Birmingham B42 2SU, UK. E-mail: elaine.denny@uce.ac.uk pain was found to limit sexual activity for the majority of the sample, with a minority ceasing to be sexually active. Lack of sexual activity resulted in a lowering of selfesteem and a negative effect on relationships with partners, although the experience differed between younger and older women.

Discussion and conclusions The experience of dyspareunia is a significant factor in the quality of life and relationships for women living with endometriosis. For most of the women in the study it was very severe and resulted in their reducing or curtailing sexual activity. Qualitative research can produce salient data that highlight the impact of dyspareunia on self-esteem and sexual relationships.

Keywords dyspareunia, endometriosis, qualitative research, quality of life, sexual relationships

J Fam Plann Reprod Health Care 2007; 33(3): 189-193

(Accepted 12 October 2006)

\section{Key message points}

- Dyspareunia is a more common symptom of endometriosis than previously reported.

- The experience of dyspareunia has an impact on quality of life and relationships for women.

- The importance placed upon the consequent avoidance of sexual activity varies among women.

been a common finding in previous studies on endometriosis and chronic pelvic pain. ${ }^{4-8}$ Ferraro et al.$^{4}$ used a sexual function questionnaire in order to characterise sexual functioning among women, and found that women with endometriosis experienced higher prevalence of deep dyspareunia than controls (60.6\% of the sample compared with $34.9 \%$ of controls), and women with disease of the uterosacral ligaments had higher prevalence than those with endometriosis in other sites. Intensity of pain in Ferraro et al.'s study was assessed using a visual analogue scale. The same authors also found that women with endometriosis have frequently experienced deep dyspareunia throughout their entire sexually active lives.

Fauconnier et al. ${ }^{5}$ utilised medical records and descriptions of the findings at the time of surgery in order to gauge the relationship between the anatomic location of deeply infiltrating endometriotic lesions and nature and severity of pain symptoms. The retrospective data analysis of Fauconnier et al.'s study found that $78.6 \%$ of women experienced dyspareunia, and it also showed that deep dyspareunia was associated with endometriotic lesions in the uterosacral ligaments. This finding was also reported by Porpora et al. 6 in their study considering the correlation between endometriosis and pelvic pain, but Vercellini, ${ }^{7}$ who considered whether prevalence and severity of pain are related to endometriosis stage and site, found that deep dyspareunia was associated more with vaginal endometriosis. Most of the current literature does not, however, have dyspareunia as its primary focus, but 
Table 1 Characteristics of the study sample

\begin{tabular}{ll}
\hline Characteristic & Value \\
\hline Average age in years (range) & 31 (19-44) \\
Socioeconomic status & 27 \\
$\quad$ Social class 1-3 & 3 \\
$\quad$ Social class 4-5 & 20 \\
Marital status & 10 \\
$\quad$ Married/cohabiting & 30 \\
Single & 0 \\
Sexual orientation & 11 (plus two pregnant \\
$\quad$ Heterosexual & at interview) \\
Homosexual & $1-3$ \\
Women with children ( $n$ ) & 27 \\
Parity (range) & 1 \\
Ethnicity & 1 \\
$\quad$ White British & 1 \\
Afro-Caribbean British & 5.65 (1-18) \\
Indo-Caribbean & \\
South American Indian & \\
Average time from symptoms to & diagnosis in years (range)
\end{tabular}

research into other aspects of endometriosis frequently finds that deep dyspareunia is a common theme in the experience of endometriosis. For example, Momoeda et $a l .8$ found that the frequency of dyspareunia increased with disease stage in women with endometriosis whose primary complaint was either chronic pelvic pain or infertility.

All of the studies cited above used quantitative methodology to correlate dyspareunia with the site of endometriosis. One of these studies was also retrospective and utilised medical records in order to obtain data. ${ }^{5}$ The study reported here is primary research, and although primarily qualitative in order to assess how much of an impact the experience of deep dyspareunia has on the quality of life in women with endometriosis, it also gathered quantitative data to gauge the extent of the problem.

\section{Methodology \\ Sample}

The sample was recruited from women attending a dedicated endometriosis outpatient clinic. The sole criterion for inclusion was laparoscopically diagnosed endometriosis, and this was confirmed in all 30 cases by the same surgeon macroscopically, and by histological examination of biopsy samples. It therefore constituted a purposive sample. Women who met the criterion were invited to participate and given an information leaflet to take home. They were telephoned a week later to confirm participation and written consent was obtained before interview. The sample size of 30 participants was arrived at by the basic qualitative research rule of data saturation; that is, recruitment to the study is suspended when no new themes emerge from additional data collected. ${ }^{9}$

Characteristics of the study sample are shown in Table 1. As can be seen from the data, the majority of the women were White British, married and from social classes 1-3.

\section{Methods}

A qualitative methodology was felt to be appropriate for the research because the aim of the study was to understand the impact of dyspareunia on women's lives rather than to generalise the findings to a wider population. Qualitative methodology offers insight into the sense people make of their world and the meaning they ascribe to events and phenomena.

A story-telling approach was adopted that enabled women to have some control over the form and content of the interviews. It allowed them to communicate the complexities of their lives and also enabled them to set parameters around what they were prepared to reveal. ${ }^{10}$ This type of qualitative research is particularly useful if experience and subjectivity are actively sought, ${ }^{11}$ and for researching sensitive issues such as sexual function. Semistructured interviews took place in a location chosen by the woman, or acceptable to her, and all the interviews were taped-recorded with the permission of the participants.

Women were initially invited to tell their story of living with endometriosis from the time at which they first experienced symptoms of endometriosis. Fourteen women spontaneously discussed painful sexual intercourse at this time. Follow-up questions by the researcher expanded on the issues raised by participants, and introduced the concept of dyspareunia to those women who had not mentioned it originally.

All interviews were transcribed verbatim. Rigour in the research process was achieved by sending women a transcript of their interview and asking them to confirm its veracity, and by both researchers being involved in the interpretation and analysis of data, and in the generation of key themes.

\section{Ethical approval}

Ethical approval for this study was obtained from the University of Central England Faculty of Health Ethics Committee and South Birmingham Research Ethics Committee.

\section{Analysis}

As a storytelling approach was utilised for the collection of data, narrative analysis was considered most appropriate, the specific model used being thematic analysis. ${ }^{9}$ This began with intensive reading and re-reading of interview transcripts, which was carried out independently by both authors. It resulted in the generation of themes, and also a framework by which to classify the data. Common themes and divergent cases were elicited and explored. Rigour in the analytical process was achieved by both authors independently analysing the data and agreeing the emergent themes. Rigour was increased by the involvement of the women in the sample in confirming the veracity of data from their own interview, and agreeing the relevance of themes.

\section{Results}

\section{Reported incidence of dyspareunia}

It was found that dyspareunia was experienced by 23 $(86 \%)$ of the valid sample (i.e. those 27 women out of the total study cohort who were sexually active) (Table 2). Eighteen $(69 \%)$ of these women also experienced pain for several hours following sexual intercourse. The majority of the women experienced pain during deep penetration, and 18 of them avoided sex because of pain. Although in longterm relationships, five women had ceased to be sexually active altogether. However, three women experienced pain but did not avoid intercourse, either because a change in position would help alleviate the pain, or because their desire for a pregnancy motivated them to endure the pain.

Table 2 Reported incidence of dyspareunia ( $n=27$ sexually active women)

\begin{tabular}{lll}
\hline Experience & $\boldsymbol{n}$ & $\%$ \\
\hline Deep dyspareunia & 23 & 86 \\
Pain after intercourse & 18 & 69 \\
Avoidance of intercourse & 18 & 69 \\
Not sexually active due to pain & 5 & 19 \\
\hline
\end{tabular}


None of the three women who were not sexually active reported any previous experience of dyspareunia. The effect that dyspareunia had on self-esteem and on relationships is discussed below.

\section{Effect on quality of life}

Salient data were generated relating to the impact of dyspareunia on a woman's quality of life and relationships. The main themes to emerge were the experience of pain, the effect on self-esteem, and the relationship with their partner. As recruitment to the study was purposive, quantifying the qualitative data is not considered appropriate. An indication of frequency is given in some instances, although the main purpose of the quotations is to illuminate the themes and provide insight into the impact of dyspareunia on the lives of women with endometriosis. ${ }^{9}$

\section{The experience of dyspareunia}

The experience of dyspareunia was extremely distressing for many women in this study. The majority of women also experienced pain in the hours or days following intercourse, and some found this type of pain more difficult to cope with than pain at the time of intercourse. For example, one woman described her experience as follows:

Researcher: "Do you get pain on intercourse?"

Participant: "Yes severe. Quite severe."

Researcher: "Just on intercourse or does it last afterwards?"

Respondent: "Afterwards, the following day as well. I have the cramps all day so obviously it puts me off."

Researcher: "I was going to ask have you avoided sex because of the pain?"

Respondent: "I have done. I mean [my baby's] father, like, he comes over at weekends, we haven't slept together for seven months near enough. We did a few days ago but like today I'm, you know, it feels like I'm bloated and I'm sore and you know I could have done without this today. It took it out of me." [18]

Some women would begin sexual intercourse with their partner, but this would sometimes have to be curtailed or the pain endured, as these women describe:

"The intercourse will start but I have to say 'no', and then there's other times when I'll just suffer in silence.” [19]

"You're with the person you love and ... You're having sex and you have to stop because it starts hurting." [17]

There was, however, diversity in the way in which women dealt with dyspareunia. As was stated previously, for some women the desire for a pregnancy would lead them to endure pain, and for others a change in position would enable them to engage in sexual activity.

Endometriosis had had such a profound effect on all aspects of life that some women were reluctant to curtail penetrative sexual activity as well.

"We've had to sacrifice quite a bit because of my health and [sexual intercourse] is one thing I really don't want to lose. That's one way I can be close to my boyfriend." [24]

However, for some women pain during or following sexual intercourse had resulted in them ceasing to engage in penetrative sex altogether, as discussed in the next section.

\section{Self-esteem}

All of the women who experienced dyspareunia experienced a negative effect on their self-esteem. This was demonstrated by women's reports of feeling unfeminine or unattractive when they were not able to engage fully in sexual intercourse. Many used the words guilty or inadequate in describing their feelings regarding the avoidance of sex, as in these reported quotes.

"I felt guilty because it wasn't fair on [my husband], but I felt relieved because by that time my life was so bound up with being in pain that I would do anything to avoid it." [1]

"I felt guilty and inadequate because I didn't want sex, and my only way of dealing with it has been to cut off and I've felt that I blame myself, and feel inadequate." [4]

The effect on self-esteem was particularly acute for those who had ceased to be sexually active. In the next quote, a young woman describes her feelings on not being able to engage in sexual activity.

"I am 22 years old. I should be having a really great relationship, but unfortunately I can't. I feel guilty even though my partner is very supportive I still feel dreadful. I don't feel particularly interested in a lot of sexual activity, because it could lead to sex and I really don't want it to, and so I stop myself. In the past it's built up quite a lot of barriers, even with affection. If I could have one thing, even with the pain, it would be that." [5]

Older women, too, regretted what they felt they had missed out on sexually.

"I feel as though I missed a hell of a lot in my life. I don't feel like that side of it has ended, it never got started I don't think ... I don't see why I shouldn't have that part of my life really." [29]

However, as will be seen in the next section, the importance placed on the lack of sexual activity varied for the women in the study, and in the reports of their partners' reactions.

\section{Relationships}

Most women described their partners as very supportive. Indeed, many of the women who had avoided sex prefaced any remarks about the effect on relationships by saying how good or understanding their partner was. However, when asked specifically about the effect of endometriosis on their relationship a number of women spoke of the tensions and arguments caused by the lack of sexual relations, as with this first quote.

"He has been great, really excellent. There are times when there is friction because it does get to him, understandably." [5]

Partners were also reported as feeling rejected by the lack of sexual activity.

"He feels rejected. You know he tries to put a brave face on it. He tries to be understanding, but he is a bloke. Yes he feels totally rejected.” [28]

"My husband takes it personally and thinks it's him though he knows that I've got this problem." [25]

Younger women in less established relationships in particular felt that lack of sexual activity jeopardised the relationship:

"It causes arguments obviously. I mean he doesn't understand that I get frustrated as well but I'd rather just forget about it than go through with the pain I suppose." [18]

"I do get worried that he's going to go off and meet someone who can give him a lot more than I can." [15] 
However, the importance that was placed on engaging in penetrative sexual intercourse varied, and older women were often more philosophical, and spoke of other qualities in their relationship, and alternatives to penetrative sex.

"He would say 'If it's going to be painful let's not even go there' in a 'don't worry about it' kind of way, and there's other things apart from sex, and there's other ways apart from penetrative sex." [4]

"I'm one of those people that can go without [sex] to be honest ... We just make love as and when we do, but there's no pressure there." [24]

Of the 30 women involved in the study, five reported that a relationship had broken up because of dyspareunia and the avoidance of sexual intercourse, including the relationship described by participants 5 and 18 above.

\section{Discussion}

Sexual intimacy is a fundamental aspect of humanity, and the inability to engage in sexual activity in a fulfilling way has a profound effect on self-esteem and on relationships with partners. ${ }^{3}$ The experience of dyspareunia is a significant factor in the quality of life of women who are living with endometriosis. The study reports graphic accounts of women's experience of living with dyspareunia caused by endometriosis. It also considers pelvic pain following intercourse, and the impact of sex avoidance on self-esteem and relationships. In doing so we have extended the previous quantitative research on the experience of dyspareunia in women with endometriosis.

The sample for the study was predominantly White British, married and from social classes 1-3. This is typical of the clinic, although not of the geographical catchment area in which it is situated. The reasons for this are unclear, but may reflect class and cultural differences in perceptions of menstruation and menstrual pain.

This study found a higher incidence of dyspareunia than previously conducted research ${ }^{4-6}$ although it is possible that other studies have defined and diagnosed symptoms differently. It may be because the study was conducted in a dedicated endometriosis clinic, and it is likely that more complex cases are referred there, although it has not yet been established that women who have more complex levels of disease definitely have a higher incidence of dyspareunia.

It could also be reflective of the methodology, and the storytelling approach that was utilised in this study. Women were encouraged to speak about all aspects of their pain, both in the clinic and during the research interviews. They were not rushed, and were given time and space to discuss their condition in whatever way they felt comfortable. Furthermore, the majority of interviews were conducted in the participant's home and they may have felt more relaxed and in control of the situation. The use of quantitative quality of life tools in a clinic setting, as was carried out in previous research, ${ }^{4-8}$ may not have been conducive to imparting sensitive information.

Dyspareunia was a common factor for 23 of the women in the study. However, as detailed within the previous sections, there was diversity in the way it was experienced. For most of the women it was very severe, and resulted in their reducing or curtailing sexual activity. The remaining women coped with the pain, either because their wish for a pregnancy outweighed their experience of pain, or because of the importance attached to physical intimacy. Some women worked out strategies to deal with the pain by avoiding the activities that produced pain, or by finding sexual positions that were more comfortable. Although some of the sample felt a profound sense of loss at the lack of sexual intimacy, others revealed that it was not of that great importance to them, and that other aspects of their relationship were valued more highly.

There were limitations to the study. Women were asked about the existence of dyspareunia and some described the pain that they experienced. Women were not, however, asked to quantify the pain, and it is likely that there was a difference in intensity, which has not been explored in this study. It is also difficult to differentiate between the effect of dyspareunia on relationships and that of pelvic pain, which has also been shown to have a detrimental effect. ${ }^{12}$ A larger, powered, quantitative sample is needed to be able to control for these variables.

The strength of this study lies in the richness of the data obtained from the interviews in which women graphically describe the impact of dyspareunia on their lives. These data supplement quantitative data of previous studies, ${ }^{4-8}$ which demonstrated the extent of deep dyspareunia in women with endometriosis, but not the impact on quality of life and relationships.

\section{Conclusions}

The main finding of this study is that dyspareunia is more commonly experienced than previous research would suggest, and that pain for some hours following intercourse is also a frequent occurrence. This has caused the majority of women in the study to avoid engaging in sexual intercourse, and in some cases to cease sexual activity altogether. Although the extent varied among women, the research found a profound effect of dyspareunia on participants' self-esteem and relationships with partners.

The significance of deep dyspareunia in terms of severity and frequency in patients suffering from endometriosis should be borne in mind when therapeutic options, such as surgical excision of the disease, are offered. Deep dyspareunia is an important symptom that must be addressed when evaluating the efficacy of treatment during follow-up of these patients in order to reduce the psychosexual sequelae of this disease.

In the clinic from which these data were collected patients are offered psychosexual counselling as part of a holistic treatment package. However, many clinics, and particularly primary care settings, do not have this facility. However, it is obviously a facility that should be offered when dealing with endometriosis patients and needs to be considered as an essential component when setting up an endometriosis treatment service.

\section{Authors' note}

In the quotations, the numbers in square brackets are the participant identification numbers.

\section{Acknowledgements}

The authors would like to thank the R\&D committee of Birmingham Women's Hospital for a grant towards this project. They are also grateful to the 30 women who agreed to take part in the research, and who shared their stories with them.

\section{Statements on funding and competing interests}

Funding The study was part funded by a grant from Birmingham Women's Hospital.

Competing interests None identified.

References

1 Society for Gynecologic Investigation 2002 Conference Report. Endometriosis: aetiology and pathophysiology. http://www. medscape.com/viewarticle/431591_3 [Accessed 10 April 2006].

2 Danewood M, Kresch AJ, Metzger D, Begany T. Current approaches to endometriosis. Patient Care 1997; 31: 34-43.

3 Campbell C, Monga A. Gynaecology by Ten Teachers (17th edn). London, UK: Edward Arnold, 2000.

4 Ferraro S, Esposito F, Abbamonte LH, Anserini P, Remorgida V, Ragni N. Quality of sex life in women with endometriosis and deep dyspareunia Fertil Steril 2005; 83: 573-579.

5 Fauconnier A, Chapron C, Dubisson J-B, Vieira M, Bousset B, 
Bréart G. Relation between pain symptoms and the anatomic location of deep infiltrating endometriosis Fertil Steril 2002; 78: 719-726.

6 Porpora MG, Koninckx PR, Piazze J, Colagrande S, Cosmi EV. Correlation between endometriosis and pelvic pain. $J A m$ Assoc Gynecol Laparosc 1999; 6: 429-434.

7 Vercellini P. Endometriosis: what a pain it is. Semin Reprod Endocrinol 1997; 13: 251-261.

8 Momoeda M, Taketani Y, Terakawa N, Hoshiai H, Tanaka K, Tsutsumi $\mathrm{O}$, et al. Is endometriosis really associated with pain?
Gynecol Obstet Invest 2002; 54(Suppl. 1): 18-21.

9 Bryman A. Social Research Methods. Oxford, UK: Oxford University Press, 2001.

10 Graham H. Surveying through stories. In: Social Researching: Politics, Problems, Practice, Bell C, Roberts H (eds). London, UK: Routledge \& Kegan Paul, 1984; 104-204.

11 Bowling A. Research Methods in Health (2nd edn). Maidenhead, UK: Open University Press, 2002.

12 Denny E. Women's experience of endometriosis. J Adv Nurs 2004; 46: 641-648.

\title{
Sexual health networks: linking providers for improvement
}

\author{
Paula Baraitser, Gary Alessio, Michael Brady
}

\section{Why was change needed?}

The National Health Service (NHS) is changing. New management structures and financial pressures have put sexual health services at risk. Commissioners are as dismayed as service users by the numerous providers in the world of sexual health and find effective planning in this context difficult. Services that work together are stronger and speak louder to commissioners.

\section{How is the new service different?}

A network is a set of autonomous organisations that come together to reach goals that no one organisation can reach separately. They may collaborate within a formal structure with clear lines of accountability or an informal one based on close working relationships.

Many areas have already set up sexual health networks in response to specific local situations. Some focus on genitourinary medicine (GUM) and HIV while others focus on sexual and reproductive health services, health promotion or the teenage pregnancy strategy. The Lambeth and Southwark Sexual Health Network (LASSHNET) is one example of a sexual health network working to link service providers and help them speak to commissioners.

\section{How is the new service organised?}

LASSHNET emerged from the work of the Lambeth and Southwark Sexual Health Modernisation Programme. LASSHNET is a very broad organisation with clinicians and senior managers from sexual and reproductive health care, GUM, health promotion, public health, voluntary sector organisations, community pharmacy, general practice and commissioning. It has a full-time network development manager and 1 day per week each from two clinical leads.

The work of the Modernisation Programme that LASSHNET is now taking on includes demand and capacity mapping, waiting and transit times monitoring and a mystery shopper programme. The programme has generated new roles for community pharmacy and a new model of sexual health service provision. A key message

\section{J Fam Plann Reprod Health Care 2007; 33(3): 193}

Lambeth and Southwark Sexual Health Network, London, UK Paula Baraitser, MD, MFFP, Clinical Lead

Gary Alessio, BA, Network Development Manager

Michael Brady, MRCP, DipGUM, Clinical Lead

Correspondence to: Dr Paula Baraitser, Lambeth and Southwark Sexual Health Modernisation Programme, Masters House,

Dugard Way (off Renfrew Road), London SE11 4TH, UK.

E-mail: paula_baraitser@mac.com from this programme is that working across the whole system of sexual health service provision brings advantages.

\section{How does the new service work in practice?}

LASSHNET has functioned effectively as part of the Modernisation Programme which organised meetings, paid for cover to ensure attendance by senior clinicians/managers and provided a link between LASSHNET and senior management in all local Trusts.

\section{What benefits does the new service offer?}

Potential benefits of collaboration include:

- Consistent clinical protocols and agreed referral pathways

- Joint undergraduate and postgraduate training programmes

- Joint strategies for service monitoring and evaluation

- Coordinated recruitment and staff induction processes

- New ways of working that cut across traditional clinical boundaries

- An integrated approach to developing and funding the whole system of sexual health service provision.

For providers of community sexual and reproductive health services engagement with local networks should be a priority. A co-ordinated network of providers with clear messages will engage commissioners and lead to effective planning.

\section{The future}

LASSHNET has developed in ideal circumstances. However, the Modernisation Programme ceases in December 2007 and LASSHNET must now plan for sustainability and meet the challenges faced by sexual health networks across the country. The most important of these is finding an organisational home and funding for managerial and clinical support.

The learning from cancer networks is that LASSHNET must accept complexity to survive. Networks may be accountable to different organisations for specific aspects of their work, their staff may be employed by different Trusts and they may get funding from a wide variety of sources. But, paradoxically, as the organisations around them reconfigure, the collaborations that make up the network may provide some much needed continuity.

\section{Author's note}

The Lambeth and Southwark Sexual Health Modernisation Programme is a 3-year service improvement programme that aims to redesign sexual health services starting from the perspective of the service users. It is funded by the Guys and St Thomas' Charity. 